\title{
Kernos
}

Revue internationale et pluridisciplinaire de religion grecque antique

$25 \mid 2012$

Varia

\section{Denver GRANINGER, Cult and Koinon in Hellenistic Thessaly}

\section{Stefano Caneva}

Édition électronique
URL : http://journals.openedition.org/kernos/2059

DOI : 10.4000/kernos.2059

ISSN : 2034-7871

\section{Éditeur}

Centre international d'étude de la religion grecque antique

\section{Édition imprimée}

Date de publication : 26 octobre 2012

Pagination : 356-358

ISSN : 0776-3824

Référence électronique

Stefano Caneva, "Denver Graninger, Cult and Koinon in Hellenistic Thessaly », Kernos [En ligne], 25 |

2012, mis en ligne le 01 octobre 2012, consulté le 21 septembre 2020. URL : http://

journals.openedition.org/kernos/2059; DOI : https://doi.org/10.4000/kernos.2059

Ce document a été généré automatiquement le 21 septembre 2020.

Kernos 


\title{
Denver GRANINGER, Cult and Koinon in Hellenistic Thessaly
}

\author{
Stefano Caneva
}

\section{RÉFÉRENCE}

Denver GRANINGER, Cult and Koinon in Hellenistic Thessaly, Leiden, 2011.1 vol. $16 \times 24 \mathrm{~cm}$, X+210 p. (Brill Studies in Greek and Roman Epigraphy). ISSN : 1876-2557. ISBN :

978-90-04-20710-3.

1 Le livre de Denver Graninger (D.G.), issu de la révision de sa thèse doctorale présentée en 2006 à la Cornell University, apporte via les sources épigraphiques une contribution variée à l'étude de l'histoire politique et culturelle de la Thessalie ancienne. Le cœur de cette recherche est constitué par l'analyse de la fonction des sanctuaires et des calendriers thessaliens dans l'organisation administrative de la région aussi bien que dans la constitution d'une identité pan-thessalienne, avec une attention particulière pour la période 196-27 av. J.-C. Dans ce but, l'A. offre aussi un profil historique des événements politiques et militaires concernant les nombreux ethnê thessaliens et leurs systèmes associatifs et administratifs, de l'époque archaïque au début du principat.

2 Un long excursus historiographique (Chapitre I : Thessalian Histories, p. 7-42) permet en effet de passer en revue les rapports entre les différents ethnê de la région, encadrés entre hégémonie des Thessaloi et autonomie des peuples perioikoi, depuis leurs origines mytho-historiques jusqu'à la réforme des critères d'assignation des votes à l'Amphictionie de Delphes sous Auguste, qui marque la fusion définitive des peuples de la région sous l'ethnique commun de Thessaloi.

3 Le deuxième chapitre (The Federal Sanctuaries, p. 43-86), constituant la première section de travail, analyse les documents concernant les deux sanctuaires pan-thessaliens où, à partir de la grande réforme réalisée par T. Quinctius Flamininus en 196, la Ligue thessalienne publia ses décrets : il s'agit du sanctuaire d'Athéna Itônia près de Philia, en Thessalie occidentale, et de celui de Zeus Eleutherios dans la capitale Larissa. 
L'A. discute la fonction pan-thessalienne des deux sanctuaires et les raisons de leur choix pour représenter l'unité de la Ligue, en observant les démarches différentes qui les concernent. D'une part, l'élévation à niveau pan-régional du culte d'Athéna Itônia, tout en s'appuyant sur des cultes précédents auxquels des observateurs non thessaliens pouvaient déjà reconnaître une importance régionale, ne se serait véritablement réalisée, selon l'analyse soigneuse qui est menée, qu'après la réorganisation fédérale achevée par Flamininus. Dès lors, le sanctuaire de Philia aurait été associé à l'identité thessalienne à cause de sa position dans une région, celle de la Thessalie occidentale, traditionnellement disputée entre Thessaliens et Macédoniens, et en plus identifiée par les récits mythiques comme la première aire d'établissement des Thessaliens provenant de l'Épire méridionale. D'autre part, l'institution du culte de Zeus Eleutherios et des fêtes Eleutheria se signale par sa complète nouveauté et par la signification clairement idéologique, qui remploie la mémoire des guerres médiques pour célébrer les Thessaliens, alignés du côté des Romains contre Philippe $\mathrm{V}$ pendant la $\mathrm{II}^{\mathrm{e}}$ guerre macédonienne. La deuxième partie du chapitre est dédiée aux Eleutheria et à une nouvelle évaluation des compétitions athlétiques et, en second temps, théâtrales qui y sont célébrées. À cet égard, D.G. combine la réédition des listes des vainqueurs de Larissa, qui fait l'objet de son appendice épigraphique (p. 159-182), avec la discussion de quelques aspects problématiques des concours, notamment les rapports entre Thessaliens et étrangers dans les compétitions et la nature de la 'triade' thessalienne. Ce dernier point se présente comme un trait distinctif des Eleutheria, dont la composition montre un lien particulier avec l'orgueil thessalien en matière de chevaux : on y trouve une chasse au taureau pratiquée à cheval, puis l'aphippolampas et l'aphippodromas, ces derniers étant respectivement une course à cheval avec une torche et, probablement, un spectacle de cavaliers descendant et remontant sur leur cheval au galop. On dispose à cet égard de quelques documents épigraphiques de Larisa (IG IX 2, 535-537) qui témoignent d'une signification rituelle de l'épreuve de la chasse au taureau; or, on connaît déjà le scepticisme de l'A., d'ailleurs en grande partie partagé, quant aux interprétations suggérant des échos initiatiques dans des pratiques religieuses thessaliennes ${ }^{1}$, mais le sujet aurait peut-être mérité une brève discussion.

4 Le troisième chapitre (The Thessalian Calendars, p. 87-114) aborde l'analyse des rapports entre les différents calendriers locaux et l'instauration d'un calendrier commun de la Ligue, dont les origines (soit que l'on propose une dérivation du calendrier de Larissa, soit une création hybride conçue dès le début comme pan-thessalienne) pourraient dater de la grande réforme de Flamininus. Selon l'A., les délais d'acceptation de ce nouveau calendrier constitueraient autant de témoignages de la résistance des communautés locales face à l'intégration pan-thessalienne, voire à la perte d'autonomie dans la réglementation du temps et des activités religieuses qui y sont liées.

5 Le quatrième chapitre (International religion, p.115-151) complète cette analyse en ajoutant une perspective extérieure, celle de l'évolution des critères d'assignation des hiéromnémons au conseil amphictionique. Cette perspective permet de détecter une coïncidence approximative entre l'intégration dans la Ligue thessalienne et l'emploi de l'ethnique des Thessaloi au niveau panhellénique. On peut en conclure que les démarches administratives de la Ligue ont contribué à la construction d'une identité régionale dans plusieurs contextes politiques et religieux (cf. p. 144-151 pour les 
dédicaces et l'envoi de theôroi au niveau international), mais qu'elles n'ont pas formé une voix unique dans ce processus.

6 Le chapitre conclusif (Conclusion and Postscript: Ainian Futures, p. 153-158) offre un aperçu des développements postérieurs des rapports entre autonomie locale et identité régionale à l'époque impériale, à travers un bref cas d'étude concernant l'histoire de la ville d'Ainis.

7 En général, même si l'on constate parfois des lacunes dans la bibliographie non anglophone ${ }^{2}$, l'analyse des enjeux politiques et idéologiques du dossier thessalien menée par l'A. est soigneuse et bien menée. En outre, on constate avec satisfaction que l'approche de l'A. s'éloigne de toute attitude primitiviste, visant à projeter ce que l'on connaît à travers les sources plus tardives sur les époques précédentes, selon la théorie tout aussi préconçue que répandue du caractère prétendument conservateur des sociétés du nord de la Grèce. Par conséquent, l'A. est à même de proposer un cadre nuancé et historiquement varié qui vise à placer chaque cas discuté, et les hypothèses qui en découlent, dans un contexte politique, religieux et culturel aussi affiné que possible.

8 On voit pourtant bien que les pratiques de gestion fédérale des sanctuaires et des concours, dont nous informent les sources, n'éclairent pas beaucoup les cultes au sens propre, à savoir les pratiques rituelles associées aux sanctuaires et aux fêtes qui y sont célébrées (cf. p. 77) : ceci tout simplement parce que la nature des sources - des décrets de la Ligue thessalienne et des listes des vainqueurs des Eleutheria - n'envisagent pas ce thème dans leurs communications. De la même façon, l'analyse des systèmes gérant les délégations des différents ethnê de Thessalie dans le conseil de l'Amphyctionie peut difficilement aboutir à une évaluation de l'identité religieuse des peuples concernés. Elle contribue plutôt à éclairer un thème différent : les manières dont la Ligue adapte et fait fonctionner les mécanismes communs de gestion du temps (les calendriers) et les relations avec les sanctuaires en regard de la création de lieux et d'occasions de représentation fédérale.

9 Ces observations nous amènent à considérer la partie peut-être la moins convaincante de cet ouvrage, à savoir le chapitre 3 sur les calendriers thessaliens. Une précision se révèle nécessaire à ce propos: on n'entend pas mettre en question le travail mené sur le dossier épigraphique attestant les noms des mois, les influences entre les calendriers des différentes poleis et le processus menant à l'affirmation du calendrier panthessalien, mais plutôt la tentative, poursuivie par l'A., de détecter les cultes thessaliens à travers les noms des mois. Il s'approprie ici (p. 89-95), de manière trop mécanique, une étude de Nilsson sur la relation entre les noms des mois grecs et leurs fêtes religieuses ${ }^{3}$ pour proposer que l'emprunt des mois entre ethnê thessaliens doit avoir comporté aussi un transfert de fêtes et, en conséquence, une révision du rythme et des contenus de la vie religieuse des cités. L'échec de cette méthode se relève dans la fréquence des expressions qui démontrent l'inadéquation de la question posée au dossier documentaire ('unattested', 4 occurrences aux p. 100-105; 'unknown', 'nonexistent', 'little indication', 'no explicit evidentiary connection', 'uncertain relationship', une fois chaque expression; 'less clear' à la p. 112), problème que l'A. luimême est forcé de reconnaître lorsqu'il observe (p. 114) que « there is no evidence that the new month festivals were widely celebrated in newly Thessalian territory (or celebrated at all - an objection which, we must admit, can be made of the tetrads as well) ». On peut remarquer à cet égard que cette équation statique entre emprunts de 
noms des mois et de fêtes se révèle un outil heuristique contraire aux scrupules de contextualisation que l'A. adopte par rapport à l'analyse des processus d'emprunt, de révision et d'invention de traditions politiques aussi bien que religieuses.

D.G. ouvre son ouvrage sur la considération que l'étude de la religion thessalienne a souffert d'un manque d'attention dans la recherche moderne et que sa contribution vise donc à attirer l'attention sur la nécessité d'études de synthèse à ce sujet (p.5): c'est justement cet objectif de work in progress que l'on peut dire achevé dans ce livre. En revanche, il faut admettre que le domaine cultuel des fêtes et des rites demeure encore en dehors de la cible. Ce regret est partiellement compensé par l'intérêt que le livre suscite du côté de l'histoire politique et des compétitions athlétiques de l'époque hellénistique.

\section{NOTES}

1. Voir son article «Studies in the cult of Artemis Throsia ", ZPE 162 (2007), p. 151-164.

2. Par exemple, sur les revendications généalogiques de Pyrrhos (cf. p. 52-54), voir notamment S. FUNKE, Aiakidenmythos und Epeirotisches Konigtum: Der Weg einer Hellenischen Monarchie, Stuttgart, 2000. Pour Platées comme lieu de mémoire de l'autonomie grecque (p.68-70), voir L. PRANDI, Platea: momenti e problemi della storia di una polis, Padova, 1988; maintenant surtout M. JUNG, Marathon und Plataiai: Zwei Perserschlachten als "lieux de mémoire“ im antiken Griechenland, Göttingen, 2006.

3. M.P. NILSSON, Primitive Time-Reckoning, Lund, 1920, p. 363-364.

\section{AUTEURS}

\section{STEFANO CANEVA}

Université de Liège 